(C) 2016 IEEE. Personal use of this material is permitted. Permission from IEEE must be obtained for all other uses, in any current or future media, including reprinting/republishing this material for advertising or promotional purposes, creating new collective works, for resale or redistribution to servers or lists, or reuse of any copyrighted component of this work in other works. 


\title{
A Single Antenna Ambient Noise Cancellation Method for In-Situ Radiated EMI Measurements in the Time-Domain
}

\author{
Marco A. Azpúrua, Marc Pous, Ferran Silva \\ Grup de Compatibilitat Electromagnètica (GCEM), Departament d'Enginyeria Electrònica (DEE) \\ Universitat Politècnica de Catalunya (UPC) \\ Barcelona, Spain \\ email: marco.azpurua@upc.edu
}

\begin{abstract}
This paper presents a single antenna ambient noise cancellation method for in-situ radiated emissions measurements performed using an entirely time-domain approach and the sliding window Empirical Mode Decomposition. The method requires a pair of successive measurements, an initial one for characterizing the ambient noise and a final one for the EMI measurement in the presence of ambient noise. The method assumes the spectral content of the ambient noise is stable between both measurements. The measured time-domain EMI is decomposed into a finite set of intrinsic mode functions. Some modes contain the ambient noise signals while other modes contain the actual components of the EMI. A brute-force search algorithm determines which mode, or combination of modes, maximize the absolute difference between the magnitude of their spectrum and the ambient noise levels for every frequency bin in the measurement bandwidth. Experimental results show the effectiveness of this method for attenuating several ambient noise signals in the $30 \mathrm{MHz}-1 \mathrm{GHz}$ band.
\end{abstract}

Keywords- Digital signal processing, electromagnetic compatibility, electromagnetic interference, electromagnetic measurements, time-domain analysis.

\section{INTRODUCTION}

In-situ electromagnetic interference (EMI) measurements are polluted by noise sources present in the same electromagnetic environment. This unavoidable electromagnetic background noise is a combination of broadcasting signals from telecommunication services and the disturbances radiated by the equipment, machinery and installations that surround the measurement antenna. The electromagnetic ambient noise is a major concern for in-situ EMI measurements because it increases the noise floor and reduces the measurement system effective sensitivity and effective dynamic range. Such degradation could make the measurement system unable to detect electromagnetic emissions generated by the EUT under evaluation.

Previously, ambient noise cancellation (ANC) techniques have been developed with the aim of undertaking the challenges imposed by the presence of ambient noise in in-situ EMI measurements. Those ANC techniques comprise alternative test methods and specific signal processing. In the following, some of those ANC techniques will be described.

The simplest and more straightforward approximation to handle ambient noise consist in performing a pair of EMI measurements, the first one with the EUT turned off and the later one with the EUT powered and active. With the information provided by the first background ambient noise measurement a few decisions can be made to improve measurement results in presence of ambient noise: 1) tune-out receiver and notch filtering narrow band ambient interferences 2) shortening the measurement distance in order to improve signal-to-noise ratio 3 ) using the signal substitution method 4) perform a linear subtraction [1]. Those frequency domain approaches to ambient noise reduction are very simple to apply but have many limitations such as: 1) effectiveness restricted to small number of narrow band signals out of the band of the EMI under assessment, 2) induce non-linear near field effects that invalidate the extrapolation of results to the original far-field distance, 3) requires additional signal generator and is again restricted to a single narrow band interference, and 4) is strictly incorrect because both measurements are not simultaneous, even if it provides approximated result in some particular cases.

Some other patented ANC methods for EMI measurement systems rely on adaptive filtering [2], [3]. Those ANC methods use simultaneous measurements performed with two identical antennas on a measurement system with two channels, one channel is expected to detect only the background ambient noise while the other one measures the combination of the EMI with the background ambient noise. Assuming the EMI signal from the EUT is uncorrelated to the ambient electromagnetic noise, and provided that the ambient noise detected by the two separated antennas is highly correlated, adaptive filter methods have been used to suppress the unwanted noise. In this regard, the Least-Mean-Square algorithm [4]-[6] and the frequency domain Overlap-Save method [7] have been used to adjust the coefficients of finite impulse response filters. Attenuation of approximately $30 \mathrm{~dB}$ has been reported in simulations and in controlled test scenarios. 
The adaptive filtering based methods of ANC have some shortcomings: 1) require the emissions from EUT to be decoupled from "only ambient noise" reference channel, 2) their effectiveness is difficult to quantify when the ambient noise share the same frequency band as the EUT emissions and 3) would require a synchronous, multiport, real-time EMI receiver.

Finally, another in-situ test method for radiated emission assessment that employs spatial spectrum estimation, adaptive beamforming, and spectrogram analysis was presented in [8]. Measurements were performed using a general purpose oscilloscope and a linear array of four omnidirectional antennas. This particular ANC method is restricted by the maximum number of antennas that can be used in the array, which is four, given by the number of channels oscilloscope. This means the algorithm is only capable of suppressing interferences from 3 different directions of arrival. On the other hand, it would be troublesome to obtain a reliable antenna factor for an array whose radiation pattern changes dynamically, thus adding significant uncertainty to the measurement results of electric field strength obtained with such a technique.

In this paper, a single antenna ANC method for in-situ radiated EMI measurements is presented. This method uses an entirely time-domain approach and the sliding window Empirical Mode Decomposition (SW-EMD) as the core processing technique. In contrast with previous ANC approaches, this method is entirely applied in the time-domain and is intended as part of a Full Time-Domain EMI (FullTDEMI) measurement system implemented with general purpose oscilloscopes. In relation with previous work, this method provides full-spectrum ANC capabilities for both continuous and transient ambient noise signals and simplifies the test setup for using a single antenna. Application cases emphasize its suitability for cancelling continuous wave and transient ambient disturbances.

The structure of this paper includes an overview of the SW-EMD (section II), a description the ANC method supported on the Full-TDEMI measurement system (section III) and presents an application example of for in-situ EMI assessment (section IV).

\section{The EMPIRICAL Mode DeCOMPOSITION}

The EMD is a heuristic method developed for analyzing nonlinear and nonstationary signals. It was introduced by Huang et al. in 1998 [9]. The main capability of the EMD is to decompose complex datasets into a finite, and often small, number of components called intrinsic mode functions (IMF) that admit well-behaved Hilbert transforms. EMD is an entirely data-driven algorithm, it does not depend on any predefined basis functions and it does not require a domain transformation.

\section{A. Overview of the EMD algorithm}

The initial step of the EMD algorithm [10] is the extraction of the extrema from signal $x(t)$ and creation of the upper envelope, $e_{\max }[x(t)]$, and the lower envelope, $e_{\min }[x(t)]$ by cubic spline interpolation of the maxima and of the minima, respectively. The mean value of the envelopes, $m[x(t)]$, is then calculated as

$$
m[x(t)]=\frac{e_{\max }[x(t)]+e_{\min }[x(t)]}{2} .
$$

An iterative process called sifting is used to identify the IMFs. In the first iteration of the sifting process, $m[x(t)]$ is subtracted from original data, that is,

$$
i m f_{1}(t)=x(t)-m[x(t)] \text {. }
$$

Then, the mean value of the envelope of $i m f_{1}(t), m\left[i m f_{1}(t)\right]$, is calculated. The sifting process is iterated until $i m f_{1}(t)$ meets the condition of an IMF $\left(m\left[i m f_{1}(t)\right] \approx 0\right)$,

$$
i m f_{1}(t):=i m f_{1}(t)-m\left[i m f_{1}(t)\right]
$$

where ":=" means it becomes.

Once the first sifting process reaches the stopping criteria, the original signal is reduced by the first mode, as given by

$$
\begin{gathered}
I M F_{1}(t):=i m f_{1}(t), \\
r_{1}(t)=x(t)-I M F_{1}(t) .
\end{gathered}
$$

The residue $r_{1}(t)$ is used as the input data for extracting the second IMF during the next sifting loop. Procedure is repeated until all the IMFs have been decomposed, which means,

$$
r_{i}(t)=r_{i-1}(t)-I M F_{i}(t)
$$

where $i$ is the index of current mode. The EMD algorithm ends when $r_{i}(t)$ has less than three extrema, because it would be impossible to construct the envelopes, or when all its points are nearly equal to zero. Therefore, the sum of all IMF components and the residue is equal to the original signal, expressed by

$$
x(t)=r_{n}(t)+\sum_{i=1}^{n} I M F_{i}(t)
$$

where $n$ is total number of the decomposed IMF.

\section{B. The Sliding Window EMD}

Many variations of the EMD algorithm have been developed. Recently, an improved version of EMD with transient decomposition capabilities was successfully used to decompose EMI complex signals [11]. However, time-domain EMI measurements require long acquisitions at very high sampling rates (several times the Nyquist limit) and analyzing EMI measurements with EMD is a resource and time consuming computational process.

In response to the aforementioned challenge, the SW-EMD was posed as by Stepien [12] as an alternative algorithm to improve computation speeds of the EMD on long datasets. The SW-EMD calculates the EMD in a relatively small window and slides this window along the time axis [12]. The particular implementation of the SW-EMD used in this work differs from original algorithm because it uses partially overlapped time windows with a length that is a function of the desired frequency resolution, according to the criteria used in [13], [14]. The EMD process is repeated for each windowed time frame and the IMFs obtained for each of those windows are then combined to reconstruct the complete set of IMF, as illustrated in Fig. 1. 


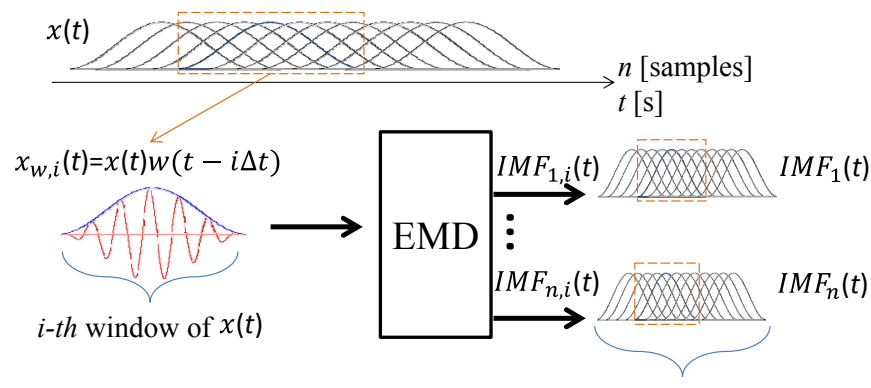

Assembling the IMFs

Fig. 1. The sliding window EMD process.

\section{A Single Antenna ANC Method}

\section{A. The Full-TDEMI measurement system}

In general terms, a Full-TDEMI measurement system is described by the block diagram shown in Fig. 2 [13]. For the measurement of radiated EMI, a broadband antenna shall be used, while for the measurement of conducted EMI corresponds either a current clamp or a line impedance stabilization network (LISN). The measured signal could be amplified or filtered if this provides better sensitivity. In the analog-to-digital converter (ADC), the full spectrum signal is digitized and stored in the time-domain. Finally, the amplitude spectrum is computed via the spectral estimation techniques.

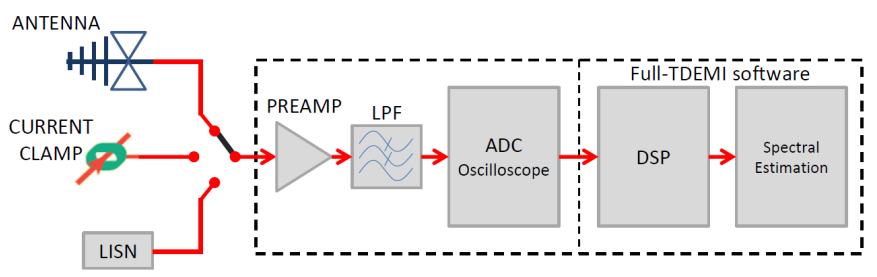

Fig. 2. The Full-TDEMI measurement system block diagram.

\section{B. The measurement setup and procedure}

With regard to the measurement setup, the general guidelines for in-situ measurements of radiated EMI produced by physically large equipment given in the CISPR TR 16-25:2008 technical report [15] shall be followed.

Additionally, the measurement procedure for ANC requires a pair of successive measurements. The first measurement is used for characterizing the ambient noise, $A N(t)$, and shall be performed with the EUT turned off. Then the measurement is repeated with the EUT turned on and without changing any of the elements of the test setup. Finally, both measurements shall be processed by the following ANC algorithm.

\section{ANC algorithm}

In the current subsection, a description of the ambient noise cancellation algorithm will be given. A simplified flowchart of the ANC algorithm is displayed in Fig. 3. The ANC algorithm has three major processing steps: the IMF combination, the spectral estimation and the linear search stage.

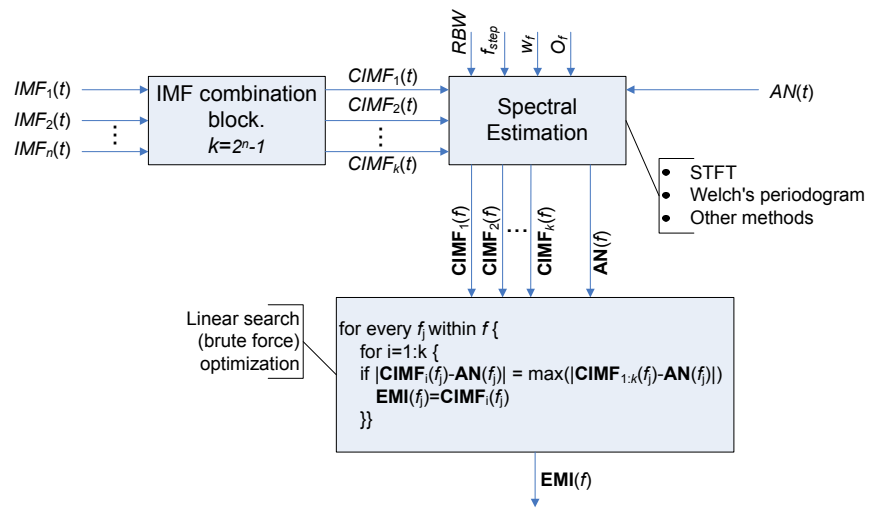

Fig. 3. ANC algorithm flowchart.

The first processing block receives the IMFs as inputs and combines them in the time domain in order to obtain the combined IMFs, CIMF. The combination rule for adding the IMFs follows a Boolean approach in where any single IMF can be (True) or not to be (False) included in a particular CIMF and it can be represented as Table I. From Table I, it is clear that the $C I M F_{k}$ corresponds approximately to the measured EMI in the presence of ambient noise if the residual is negligible.

TABLE I. CIMFS COMBINATION RULE

\begin{tabular}{|c|c|c|c|c|}
\hline \multirow{2}{*}{$C I M F_{m}$} & \multicolumn{4}{|c|}{$I M F_{i}$} \\
\cline { 2 - 5 } & $n$ & $\ldots$ & 2 & 1 \\
\hline 1 & $\mathrm{~F}$ & - & $\mathrm{F}$ & $\mathrm{T}$ \\
\hline 2 & $\mathrm{~F}$ & - & $\mathrm{T}$ & $\mathrm{F}$ \\
\hline$\ldots$ & - & - & - & - \\
\hline$k-1$ & $\mathrm{~T}$ & - & $\mathrm{T}$ & $\mathrm{F}$ \\
\hline$k$ & $\mathrm{~T}$ & - & $\mathrm{T}$ & $\mathrm{T}$ \\
\hline
\end{tabular}

The second stage of the ANC algorithm corresponds to the spectral estimation. The CIMF and the ambient noise (AN) signals are transformed into the frequency domain using the Short-Time Fourier Transform (STFT) and non-parametric methods, such as Welch's periodogram. The appropriate resolution bandwidth (RBW), frequency step size $\left(f_{\text {step }}\right)$, window factor $\left(w_{f}\right)$ and overlapping factor $\left(o_{f}\right)$ shall be configured in order to obtain results coherent with EMI receiver specifications.

The last step in the ANC algorithm processing chain is the linear search. Linear search is the simplest form of the brute force search algorithm. The linear search is used to identify, one frequency at the time, which CIMF differs the most form the ambient noise. Therefore, the estimated EMI spectrum is assembled within a "for" loop, cancelling the effect of the CIMFs that are most likely to carry the ambient noise signals.

\section{APPLICATION EXAMPLE}

In this section, an application example of the introduced ANC method for in-situ radiated EMI measurements in the time domain will be presented. Measurements were performed with a Full-TDEMI measurement system based on a Tektronix DPO5104B oscilloscope, using a sampling rate of $5 \mathrm{GS} / \mathrm{s}$ and 
a record length of $1 \mathrm{~ms}$, in the $30 \mathrm{MHz}-1 \mathrm{GHz}$ frequency band, using a $120 \mathrm{kHz}$ resolution bandwidth and a $30 \mathrm{kHz}$ step size [11], [13], [14], [16]. The test setup was placed inside a Full Anechoic Room (FAR) from which the doors were left intentionally opened in order to capture the ambient noise signals.

The source of the EMI signal was a Comparison Noise Emitter III (CNE-III) from York EMC services. A Schaffner CBL6143 bilog antenna in horizontal polarization was used. The antenna was positioned for a 3-m EUT-to-test-antenna distance. Antenna factors were not applied to the measurements. In consequence, measurement results are given in terms of the voltage measured at the antenna port.

The result of the ambient noise characterization is shown in Fig. 4. The expected signals from radio broadcasting, digital terrestrial television and mobile communication services are clearly identifiable.

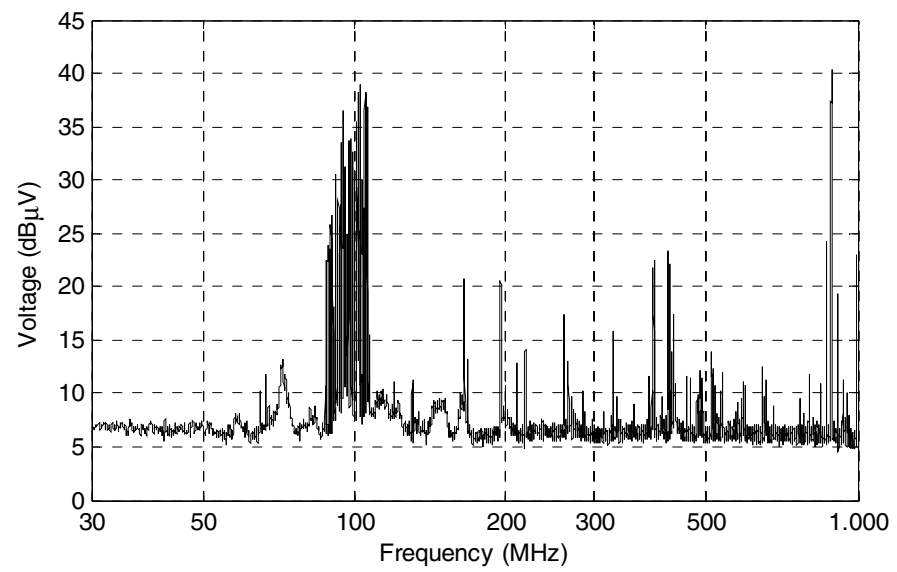

Fig. 4. In-situ background ambient noise characterization.

Next, the CNE-III is turned on and the EMI signal is measured in the presence of ambient noise. The results are shown in Fig. 5. The most noticeable aspect of Fig. 5 spectrum is the presence of a broadband noise signal. Inspecting the time domain measurements (Fig 6), a transient event was detected in the EMI measurement. In this particular example, the transient signals were intentionally generated by turning on a set of fluorescent lights in a room next to the FAR.

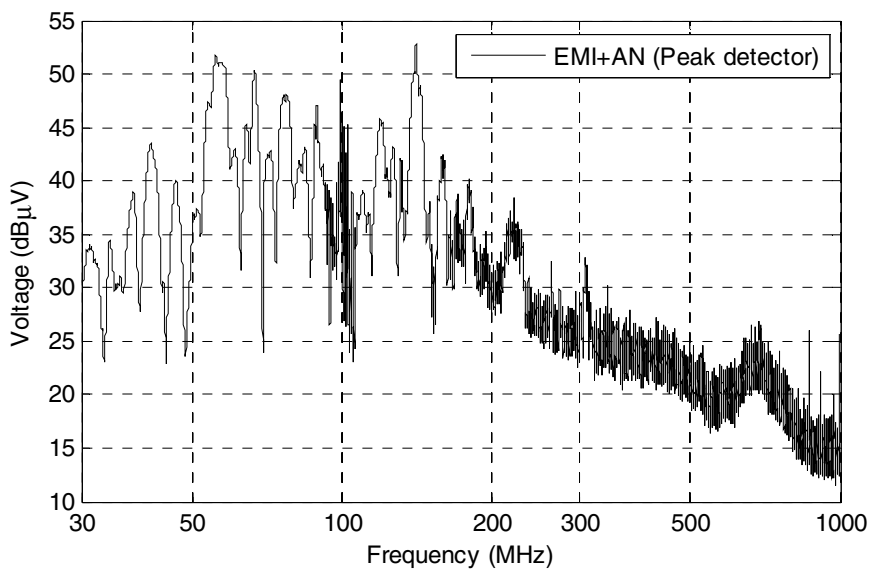

Fig. 5. EMI measurements in the presence of ambient noise.

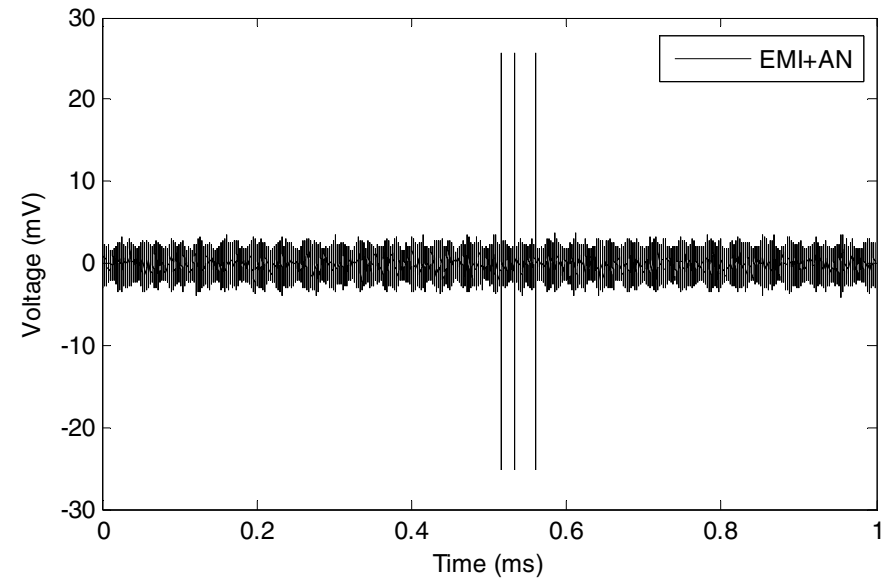

Fig. 6. Time domain EMI measurements in the presence of ambient noise.

The aforementioned transient event is part of the ambient noise signals. In order to remove the transient pulses from the EMI, techniques presented in [11] were applied.

The SW-EMD is used to decompose the measured EMI signal in the presence of ambient noise. Four IMFs were identified, as shown in Fig. 7. IMF 1 corresponds to the transient event. $\mathrm{IMF}_{2}$ includes the ambient noise signals of higher frequency. $\mathrm{IMF}_{3}$ contains most of the energy of the EMI. $\mathrm{IMF}_{4}$ contains the ambient noise signal corresponding with FM broadcasting. Since the EUT has broadband emissions in the whole frequency range, part of the energy of the EMI signal is distributed along the $\mathrm{IMF}_{2,3,4}$.
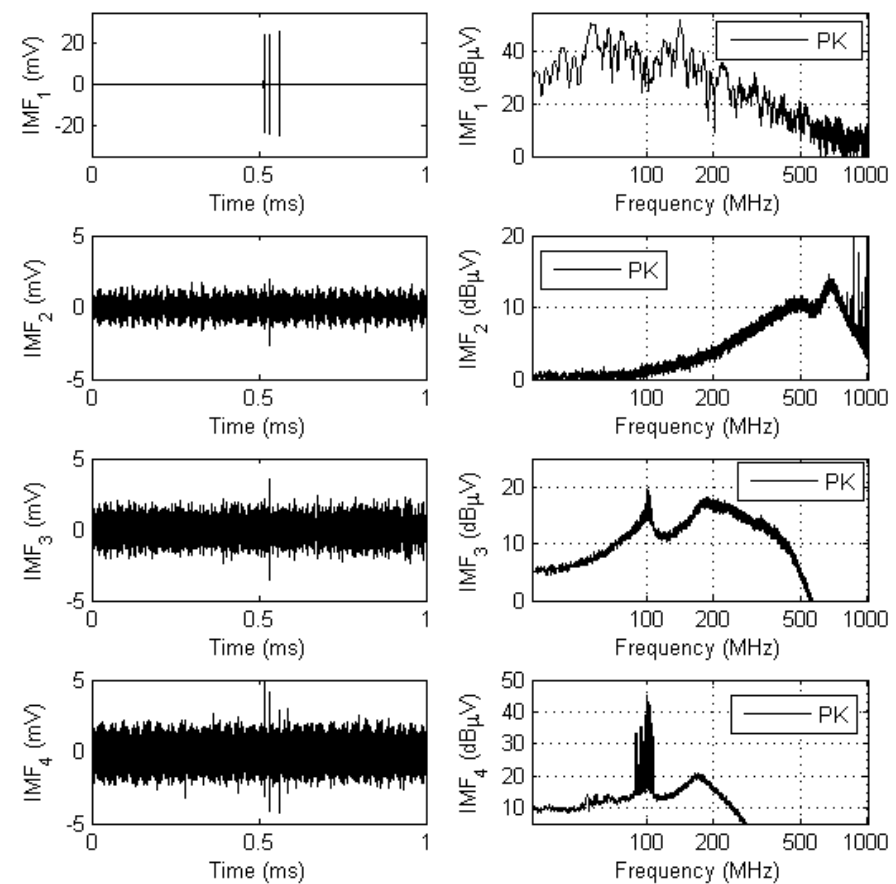

Fig. 7. IMF of the measured EMI in the presence of ambeint noise.

Finally, the EMI signal is estimated using the ANC algorithm explained in the previous section. The results are shown in Fig. 8. Several ambient noise signals have been cancelled achieving up to $25 \mathrm{~dB}$ attenuation. 
For validation purposes, the EMI measurement was repeated with the FAR doors closed. The results of EMI estimated after applying the ANC method are in excellent agreement with the EMI measurements in the absence of ambient noise. A minor level of mode mixing is evidenced in the FM broadcasting band.

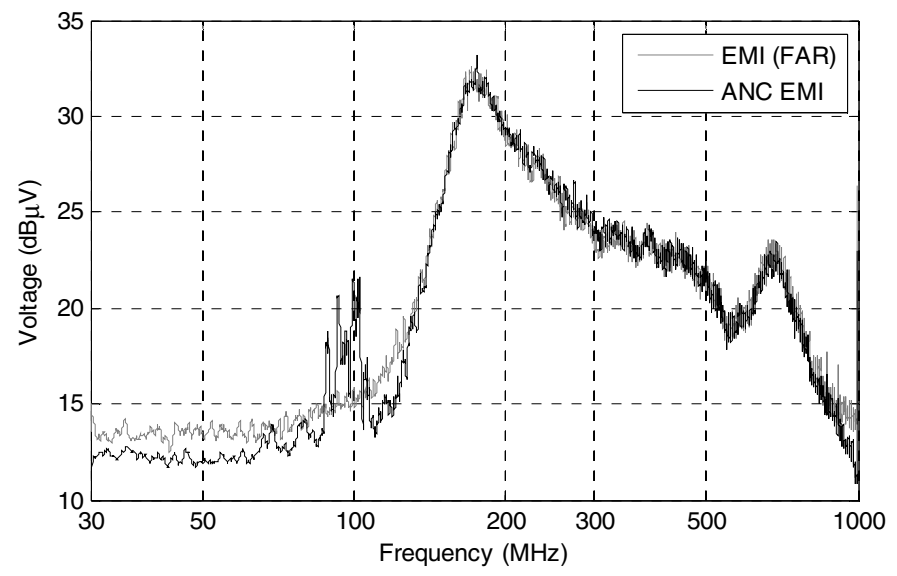

Fig. 8. Estimated EMI amplitud spectrum after ANC.

\section{DISCUSION}

The presented ANC method relies on the assumption that ambient noise remains stable (in terms of its spectral content) between the EUT being switched off, and the EUT having booted up to normal operation. This rise the concern about changes in the ambient noise caused by sporadic "pop up" signals that may appear during only one of the two required measurements, i.e. mobile radio communication signals from moving sources like police vehicles and similar.

In such situations, this method would be unable to cancel properly such irruptive "pop up" signals. As an alternative, time/frequency representations (spectrograms) of the EMI signal can be used to identify such sporadic signals. Then it would be possible to preprocess the time-domain EMI measurement (time-gating) for excluding the specific sub time-frame in which the intermittent signal occurred. Moreover, this ANC approach could also be extended for using a pair of antennas for performing ambient noise and EMI measurements simultaneously and synchronously, thus improving its capabilities for cancelling sporadic ambient noise signals.

\section{CONCLUSION}

A single antenna ANC method for in-situ EMI measurements has been introduced. The method requires a pair of measurements, one for characterizing the ambient noise and another for the EMI measurement in the presence of ambient noise. It is based on time-domain EMI decomposition performed using the SW-EMD and a brute-force search algorithm that determines which mode, or combination of modes, maximize the absolute difference between the magnitude of their spectrum and the ambient noise levels for every frequency bin within the measurement bandwidth. In comparison with other previously reported ANC methods for
EMI measurements, this ANC method simplifies the measurement setups because it uses only one antenna. Another advantage of the ANC method, in comparison with the previously published techniques, is that it allows cancelling the effect of transient ambient noise. This ANC method is performed through off-line post-processing. Experimental results show the effectiveness of this method for attenuating several ambient noise signals in the $30 \mathrm{MHz}-1 \mathrm{GHz}$ band.

As a final remark, it is important to realize ANC methods are not intended to replace expert judgement in complex insitu radiated EMI measurements. Aspects such as the selection of an adequate antenna location and orientation, the evaluation of multipath induced errors, the identification of sporadic "pop up" signals, among many other factors still require to be analyzed on an individual basis in order to decide the best possible course of action for the radiated emissions assessment.

\section{ACKNOWLEDGMENT}

This work was supported in part by the EURAMET IND60EMC research project (the EMRP is jointly funded by the EMRP participating countries within EURAMET and the European Union) and by the Spanish "Ministerio de Economía y Competitividad," under project TEC2013-48414-C3-3-R.

\section{REFERENCES}

[1] J. Svačina, J. Dřínovský, and R. Vídenka, "Virtual anechoic room - An useful tool for EMI pre-compliance testing," in 2007 17th International Conference Radioelektronika, 2007.

[2] M. Marino, "System and method for measuring rf radiated emissions in the presence of strong ambient signals," 2005.

[3] S. Braun, A. Frech, and P. Russer, "Method for rejection of ambient interference signal during measurement of interference emissions of electric and electronic devices, involves digitalizing signal of antenna through analog to digital converter," 2009.

[4] A. Frech, S. Limmer, and P. Russer, "Noise cancelling algorithms for FPGA-based time-domain EMI measurements in real-time," in IEEE International Symposium on Electromagnetic Compatibility, 2011, pp. 484- 488 .

[5] A. Frech, M. Klugel, and P. Russer, "Adaptive filtering for noise cancellation and signal analysis in real-time," Microwave Conference (EuMC), 2013 European. pp. 1123-1126, 2013.

[6] C. Osterwise, S. L. Grant, and D. Beetner, "Reduction of noise in nearfield measurements," in IEEE International Symposium on Electromagnetic Compatibility, 2010, pp. 171-176.

[7] A. Frech, S. Braun, and P. Russer, "Time-domain EMI measurements in the presence of ambient noise," in IEEE International Symposium on Electromagnetic Compatibility, 2009, pp. 139-142.

[8] Z.-H. Lu, J.-B. Liu, and P.-G. Liu, "A Novel Method of Ambient Interferences Suppressing for In Situ Electromagnetic Radiated Emission Test," Electromagnetic Compatibility, IEEE Transactions on, vol. 54, no. 6. pp. 1205-1215, 2012.

[9] N. E. Huang, Z. Shen, S. R. Long, M. C. Wu, H. H. Shih, Q. Zheng, N.C. Yen, C. C. Tung, H. H. Liu, N. E. Huang, Z. Shen, S. Long, M. Wu, H. Shih, Q. Zheng, N.-C. Yen, C. Tung, and H. Liu, "The empirical mode decomposition and the Hilbert spectrum for nonlinear and nonstationary time series analysis," Proc. R. Soc. London. Ser. A Math. Phys. Eng. Sci., vol. 454, no. 1971, pp. 903-995, 1998.

[10] G. Rilling, P. Flandrin, P. Gon, and D. Lyon, "on Empirical Mode Decomposition and Its Algorithms," IEEEEURASIP Work. Nonlinear Signal Image Process. NSIP, vol. 3, pp. 8-11, 2003.

[11] M. A. Azpúrua, M. Pous, and F. Silva, "Decomposition of 
Electromagnetic Interferences in the Time-Domain," IEEE Trans. Electromagn. Compat., pp. 1-8, 2016.

[12] P. Stepien, "Sliding Window Empirical Mode Decomposition -its performance and quality," EPJ Nonlinear Biomed. Phys., vol. 2, no. 1, p. 14, 2014.

[13] M. A. Azpúrua, M. Pous, and F. Silva, "A Measurement System for Radiated Transient Electromagnetic Interference Based on General Purpose Instruments," in Electromagnetic Compatibility (EMC EUROPE), International Symposium on, 2015.

[14] E. M. I. Time-Domain and I. Magazine, "M. Azpúrua, M. Pous and F.," Silva Improv. Meas. through Digit. Signal Process. Electromagn. Compat., 2015.

[15] IEC CISPR, “CISPR TR 16-2-5:2008 Specification for radio disturbance and immunity measuring apparatus and methods - Part 2-5: In situ measurements for disturbing emissions produced by physically large equipment," 2008.

[16] M. A. Azpurua, M. Pous, and F. Silva, "On the Statistical Properties of the Peak Detection for Time-Domain EMI Measurements," pp. 1-8, 2015. 